\title{
Ectonucleotidases in Müller glial cells of the rodent retina: Involvement in inhibition of osmotic cell swelling
}

\author{
Ianors Iandiev • Antje Wurm • \\ Thomas Pannicke • Peter Wiedemann • \\ Andreas Reichenbach • Simon C. Robson • \\ Herbert Zimmermann • Andreas Bringmann
}

Received: 30 May 2007 / Accepted: 10 July 2007 / Published online: 8 August 2007

(C) Springer Science + Business Media B.V. 2007

\begin{abstract}
Extracellular nucleotides mediate glia-to-neuron signalling in the retina and are implicated in the volume regulation of retinal glial (Müller) cells under osmotic stress conditions. We investigated the expression and functional role of ectonucleotidases in Müller cells of the rodent retina by cell-swelling experiments, calcium imaging, and immunoand enzyme histochemistry. The swelling of Müller cells under hypoosmotic stress was inhibited by activation of an autocrine purinergic signalling cascade. This cascade is initiated by exogenous glutamate and involves the consecutive activation of $\mathrm{P}_{2} \mathrm{Y}_{1}$ and adenosine $\mathrm{A} 1$ receptors, the action of ectoadenosine $5^{\prime}$-triphosphate (ATP)ases, and a nucleoside-transporter-mediated release of adenosine. Inhibi-
\end{abstract}

I. Iandiev $\cdot$ A. Wurm $\cdot$ T. Pannicke $\cdot$ A. Reichenbach

Paul Flechsig Institute of Brain Research,

University of Leipzig,

Leipzig, Germany

I. Iandiev

Translational Center for Regenerative Medicine,

University of Leipzig,

Leipzig, Germany

\author{
A. Wurm $\cdot$ P. Wiedemann $\cdot$ A. Bringmann $(\bowtie)$ \\ Department of Ophthalmology and Eye Clinic, \\ University of Leipzig Medical Faculty, \\ Liebigstrasse 10-14, \\ 04103 Leipzig, Germany \\ e-mail: bria@medizin.uni-leipzig.de \\ S. C. Robson \\ Department of Medicine, Beth Israel Deaconess Medical Center, \\ Harvard Medical School, \\ Boston, MA, USA \\ H. Zimmermann \\ Biocenter, J.W. Goethe-University, AK Neurochemistry, \\ Frankfurt am Main, Germany
}

tion of ectoapyrases increased the ATP-evoked calcium responses in Müller cell endfeet. Müller cells were immunoreactive for nucleoside triphosphate diphosphohydrolases (NTPDase)2 (but not NTPDase1), ecto-5'-nucleotidase, $\mathrm{P}_{2} \mathrm{Y}_{1}$, and A1 receptors. Enzyme histochemistry revealed that ATP but not adenosine $5^{\prime}$-diphosphate (ADP) is extracellularly metabolised in retinal slices of NTPDase 1 knockout mice. NTPDase1 activity and protein is restricted to blood vessels, whereas activity of alkaline phosphatase is essentially absent at physiological $\mathrm{pH}$. The data suggest that NTPDase2 is the major ATP-degrading ectonucleotidase of the retinal parenchyma. NTPDase2 expressed by Müller cells can be implicated in the regulation of purinergic calcium responses and cellular volume.

Keywords A1 receptor . Cellular swelling .

Ectonucleotidase $\cdot$ Müller glial cell $\cdot$ P2 receptor $\cdot$ Retina

\section{Introduction}

Glial cells play an active role in the regulation of neuronal activity. By release of so-called gliotransmitters, in particular glutamate and adenosine 5'-triphosphate (ATP), activated glial cells provide both excitatory and inhibitory effects on neighbouring neurons. Excitation is mediated predominantly by glutamate, whereas ATP, after extracellular conversion to adenosine, causes neuronal suppression [1-3]. In the neural retina, stimulation of Müller glial cells by receptor agonists such as ATP, dopamine and thrombin, or electrical and mechanical stimuli, triggers intracellular calcium responses associated with a release of ATP from the cells $[4,5]$. It has been suggested that ATP released from Müller cells is extracellularly converted to adenosine, which in turn 
activates A1 receptors in a subset of retinal ganglion cells, resulting in cellular hyperpolarisation [5]. In addition to the involvement in glia-to-neuron signalling, glia-derived nucleotides in the retina have autocrine effects. ATP released from retinal glial cells and subsequent activation of purinergic receptors evokes long-range calcium waves in the glial cell network [4] and inhibits the swelling of Müller cells under conditions of osmotic stress [6].

Extracellular metabolism of ATP has been implicated in the glia-to-neuron signalling in the retina [5]. It is known that Müller cells express ecto-5'-nucleotidase (CD73) [7-9], at least during the period of postnatal vasculogenesis [10]. CD73 hydrolyses nucleoside monophosphates such as AMP to the respective nucleosides [11]. However, it is unclear whether Müller cells also express ectoenzymes that degrade ATP, such as nucleoside triphosphate diphosphohydrolases (NTPDases) or ectonucleotide pyrophosphatase/phosphodiesterases (ENPPs). NTPDase1 (CD39, ectoapyrase) and NTPDase2 (CD39L1, ecto-ATPase), as well as NTPDases 3 and 8 , are capable of hydrolysing nucleoside 5 'tri- and diphosphates such as ATP and ADP, albeit with considerably different substrate preference. Whereas NTPDase1 hydrolyses ATP and ADP about equally well, NTPDase2 preferentially degrades ATP, and NTPDases 3 and 8 reveal intermediate substrate preferences $[12,13]$. In the neural retina, ADPase enzyme histochemistry and CD39 immunohistochemistry allocated NTPDase1 exclusively to the vasculature $[14,15]$. In the brain, NTPDase1 is associated with microvessels and microglial cells but not with neurons or astrocytes [16]. NTPDase2 is expressed in the germinal zones of the developing and adult brain and by subpial astrocytes [17, 18]. In the peripheral nervous system, NTPDase1 is expressed by blood vessels and NTPDase2 by various types of glial cells [19]. ENPP1 is an ectoenzyme with both 5'-nucleotide phosphodiesterase and nucleotide pyrophosphatase activity [20]. It is expressed in cell lines derived from brain glial cells and has also been allocated to brain capillaries and synaptosomal plasma membranes [12, 21-23]. The aim of our study was to investigate whether extracellular nucleotide metabolism is implicated in the inhibitory effect of ATP on the osmotic swelling of Müller cells, to determine the types of ectonucleotidases expressed by Müller cells and whether the retinal expression of ectonucleotidases is altered after transient retinal ischaemia reperfusion.

\section{Materials and methods}

Materials

Mitotracker Orange (chloromethyltetramethylrosamine) and Fluo-4/AM were purchased from Molecular Probes (Eugene,
OR, USA). Adenosine-5'-O-( $\alpha, \beta$-methylene $)$-diphosphate (AOPCP), 6- $N, N$-diethyl- $D$ - $\beta, \gamma$-dibromomethylene ATP (ARL-67156), 8-(3-chlorostyryl) caffeine (CSC), 8-cyclopentyl-1,3-dipropylxanthine (DPCPX), $N^{6}$-methyl2'-deoxyadenosine-3',5'-bisphosphate (MRS2179), $N$ nitrobenzylthioinosine (NBTI), p-nitrophenyl phosphate (pNPP), pyridoxal-phosphate-6-azophenyl-2',4'-disulfonic acid (PPADS), and all other substances used were obtained from Sigma-Aldrich (Taufkirchen, Germany) unless stated otherwise. The following antibodies were used: rabbit anti-NTPDase1 (1:200) [16], rabbit antiNTPDase2 (1:500) [17], goat anti-ENPP1 (1:200; Santa Cruz), goat anti-ecto-5'-nucleotidase (1:200; Santa Cruz), rabbit anti-P2Y 1 (1:100; Alomone), rabbit anti-A1 receptor (1:200; Santa Cruz), mouse anti-vimentin (1:200; V9 clone, Santa Cruz), mouse anti-glutamine synthetase (1:250, Chemicon) and mouse anti-cellular retinaldehyde-binding protein (CRALBP; 1:1000; Acris). The secondary antibodies were $\mathrm{Cy} 3-$ conjugated goat antirabbit immunoglobulin (Ig)G (1:400; Dianova), Cy2-coupled goat anti-mouse IgG (1:200; Dianova), Cy3-coupled donkey anti-goat IgG (1:200; Dianova) and Cy2-coupled donkey antimouse IgG (1:200; Dianova).

\section{Animals}

All experiments were performed in accordance with the European Communities Council Directive 86/609/EEC and were approved by the local authorities. Adult Long-Evans rats $(250-350 \mathrm{~g})$, NTPDase1 ( $c$ d39)-deficient mice, and wild-type mice were used. The generation of NTPDase1deficient mice from wild-type animals has been described previously [24]. Animals had free access to water and food in an air-conditioned room on a 12-h light-dark cycle. Transient retinal ischaemia was induced in one eye of the rats, whereas the other eye remained untreated and served as control (three animals). Anaesthesia was induced by intramuscular ketamine $(100 \mathrm{mg} / \mathrm{kg})$ and xylazine $(5 \mathrm{mg} / \mathrm{kg})$. The anterior chamber of the treated eye was cannulated from the pars plana with a 27 -gauge infusion needle connected to a bag containing normal saline. The intraocular pressure was increased to $160 \mathrm{mmHg}$ for $60 \mathrm{~min}$ by elevating the saline bag. The animals were killed by carbon dioxide 3 days after reperfusion, and the eyes were removed.

Müller cell swelling

To determine volume changes of Müller glial cells evoked by hypotonic stress, the somata of the cells in the inner nuclear layer of rat retinal slices were recorded. Acutely isolated slices (thickness, $1 \mathrm{~mm}$ ) were placed in a perfusion chamber and loaded with the vital dye Mitotracker Orange $(10 \mu \mathrm{M})$. This dye is selectively taken up by Müller 
cells, whereas retinal neurons, astrocytes and microglial cells remain unstained [25]. The stock solution of the dye was prepared in dimethylsulfoxide and resolved in saline. The slices were examined with a confocal laser scanning microscope (LSM) LSM 510 Meta (Zeiss, Oberkochen, Germany). Mitotracker Orange was excited at $543 \mathrm{~nm}$, and emission was recorded with a $560 \mathrm{~nm}$ long-pass filter. In the course of the experiments, the somata of Müller cells were recorded at the plane of their maximal extension.

A gravity-fed system with multiple reservoirs was used to perfuse the recording chamber continuously with extracellular solution; the hypotonic solution and test substances were added by rapidly changing the perfusate. The extracellular solution consisted of $(\mathrm{mM})$ $136 \mathrm{NaCl}, 3 \mathrm{KCl}, 2 \mathrm{CaCl}_{2}, 1 \mathrm{MgCl}_{2}, 10$ hydroxyethylpiperazine ethanesulfonic acid (HEPES) and 11 glucose, adjusted to $\mathrm{pH} 7.4$ with Tris. The hypotonic solution (60\% of control osmolarity) was made up by adding distilled water. Barium chloride was added to the extracellular solution and was preincubated for $10 \mathrm{~min}$. Blocking substances were preincubated for 15-45 min, and agonists were administered simultaneously with the hypotonic solution.

\section{Calcium imaging}

Wholemounts of the rat retina $\left(9 \mathrm{~mm}^{2}\right)$ were placed in a perfusion chamber and incubated for $1 \mathrm{~h}$ at room temperature in extracellular solution containing the calcium-sensitive fluorescence dye Fluo-4/AM $(11 \mu \mathrm{M})$. After $10 \mathrm{~min}$ of washing by continuous perfusion of extracellular solution, ATP was added by a fast change of the perfusate. ARL67156 was preincubated for 25 min. Fluo-4 was excited at $488 \mathrm{~nm}$; emission was recorded with a band-pass filter between 505 and $550 \mathrm{~nm}$.

\section{Immunohistochemistry}

Isolated rat retinas were fixed in $4 \%$ paraformaldehyde for $2 \mathrm{~h}$. After several washing steps in buffered saline, the tissues were embedded in saline containing 3\% agarose $(\mathrm{w} / \mathrm{v})$, and $60-\mu \mathrm{m}$-thick slices were cut by using a vibratome. For double labelling, the slices were incubated in 5\% normal goat or donkey serum plus $0.3 \%$ Triton X100 in saline for $2 \mathrm{~h}$ at room temperature and, subsequently in a mixture of primary antibodies overnight at $4^{\circ} \mathrm{C}$. After washing in $1 \%$ bovine serum albumin, secondary antibodies were administered for $2 \mathrm{~h}$ at room temperature. Control slices were stained without primary antibodies; no unspecific labelling was observed following incubation with secondary antibodies (not shown). Images were taken with the LSM.
Immunocytochemistry

To prepare suspensions of dissociated cells, isolated rat retinas were stored for $30 \mathrm{~min}$ in saline containing $0.4 \mathrm{mg} / \mathrm{ml}$ papain (Boehringer, Mannheim, Germany) at $37^{\circ} \mathrm{C}$. After fixation with $4 \%$ paraformaldehyde for $10 \mathrm{~min}$ and washing with saline, the tissues were triturated with a pipette until single cells were dissociated. The cells were blocked and permeabilised for $30 \mathrm{~min}$ with $5 \%$ normal goat serum and $0.3 \%$ Triton-X 100 , followed by incubation with primary antibodies for $6 \mathrm{~h}$ at $4{ }^{\circ} \mathrm{C}$. After washing in $1 \%$ bovine serum albumin in saline, the secondary antibodies were applied for $1 \mathrm{~h}$ at room temperature.

Enzyme histochemistry

Mice were deeply anaesthetised with pentobarbital $(800 \mathrm{mg} / \mathrm{kg}$ ) and perfused intracardially with phosphatebuffered saline containing heparin $(0.5 \mathrm{mg} / \mathrm{ml})$ followed by $0.05 \mathrm{M}$ cacodylate-buffered $4 \%$ paraformaldehyde, $\mathrm{pH}$ 7.4. Subsequently, eyes were removed and immersed at $4^{\circ} \mathrm{C}$ in the same fixative for $4 \mathrm{~h}$. The eyes were cryoprotected overnight with $30 \%$ sucrose and frozen in isopentane at $-80^{\circ} \mathrm{C}$. Frozen tissues were stored at $-80^{\circ} \mathrm{C}$ until sectioning. Frozen sections $(14 \mu \mathrm{m})$ were deposited on 3aminopropyl-triethoxysilane-coated slides and allowed to dry for $1 \mathrm{~h}$. The dry sections were stored at $-80^{\circ} \mathrm{C}$ until further processing. For localisation of ectonucleotidase activity, a lead phosphate method was applied, as previously described [16]. In brief, frozen sections were warmed to room temperature. The enzyme reaction was performed for $1 \mathrm{~h}$ at room temperature in a TMS-buffered substrate solution (in mM: $2 \mathrm{~Pb}\left(\mathrm{NO}_{3}\right)_{2}, 5 \mathrm{MnCl}_{2}, 2 \mathrm{CaCl}_{2}, 50$ Trismaleate, $\mathrm{pH} 7.4$, plus $0.25 \mathrm{M}$ sucrose) stabilised with $3 \%$ dextran T 250 (Roth, Karlsruhe, Germany) containing the substrates ATP, ADP or pNPP (sodium salts; $1 \mathrm{mM}$ each). In control experiments, the substrate was omitted from the incubation solution. After washing sections with demineralised water, the lead orthophosphate precipitated as a result of nucleotidase activity was visualised as a brown deposit by incubating sections in an aqueous solution of $\left(\mathrm{NH}_{4}\right)_{2} \mathrm{~S}$ $(1 \% \mathrm{v} / \mathrm{v})$.

\section{Data analysis}

To determine the extent of swelling of Müller cell somata, the cross-sectional area of Mitotracker-Orange-stained cell bodies in the inner nuclear layer of retinal slices was measured off-line using the analysis software of the LSM. Bar diagrams display the mean $[ \pm$ standard error of the mean (SEM)] cross-sectional areas of cell somata that were measured after 4-min perfusion with hypotonic solution relative to the somatic area measured before hypotonic 
challenge (100\%). To evaluate the calcium responses, the fluorescence of Fluo- 4 was normalised to the prestimulus value by calculation of the ratio $F / F_{0}$, whereby $F_{0}$ represents the baseline fluorescence before agonist application. Statistical analysis was performed using the Prism program (Graphpad Software, San Diego, CA, USA); significance was determined by Student's $t$ test or Kruskal-Wallis test followed by Dunn's comparison for multiple groups.

\section{Results}

Purinergic signalling inhibits osmotic swelling of Müller glial cells

To determine whether extracellular nucleotide metabolism is implicated in the inhibitory effect of ATP on the osmotic swelling of retinal glial cells, we investigated the alterations in size of Müller cell somata in acutely isolated rat retina slices in response to hypotonic stress. As described previously [26, 27], administration of a hypotonic solution (containing $60 \%$ of control osmolarity) did not alter the size of Müller cell somata in slices of control retinas, whereas Müller cell somata swelled in the presence of barium ions (Fig. 1a,b). Similarly, Müller cell somata in postischaemic retinas displayed hypotonic swelling. We recently showed that various purinergic receptor agonists, such as ATP, ADP and adenosine, inhibit osmotic swelling of Müller cell somata and that glutamate also inhibits the swelling via activation of a purinergic signalling cascade [6]. This signalling cascade is apparently mediated by autocrine mechanisms in Müller cells, as indicated by the lack of effect of the blocker of voltage-gated sodium channels tetrodotoxin on the action of glutamate, ATP and adenosine (Fig. 1c). The swelling-inhibitory action of glutamate and ATP were blocked by suramin and the inhibitor of $\mathrm{P} 2 \mathrm{Y}$ and distinct $\mathrm{P} 2 \mathrm{X}$ receptors, PPADS (Fig. 1d). The selective $\mathrm{P}_{2} \mathrm{Y}_{1}$ receptor antagonist, MRS2179, prevented the effects of glutamate and ATP but not of adenosine (Fig. 1e), whereas the antagonist of adenosine A1 receptors, DPCPX, prevented the effects of all three agonists investigated (Fig. 1f). The selective A2a receptor antagonist, CSC, had no effect (Fig. 1f). The data suggest that the swelling-inhibitory effects of glutamate and ATP are mediated by activation of $\mathrm{P}_{2} \mathrm{Y}_{1}$ and A1 receptors, and that the activation of $\mathrm{A} 1$ receptors occurs downstream from $\mathrm{P}_{2} \mathrm{Y}_{1}$ receptor activation. Apparently, glutamate evokes a consecutive release (or formation) of ATP and adenosine, which successively activates $\mathrm{P}_{2} \mathrm{Y}_{1}$ and $\mathrm{A} 1$ receptors in Müller cells.

Though $\mathrm{P}_{2} \mathrm{Y}_{1}$ receptors are suggested to be preferentially activated by ADP [28], there are also studies that show an activation by ATP $[29,30]$. To determine whether extra- cellular nucleotide metabolism is involved in the swellinginhibitory effect of glutamate, we tested an inhibitor of ectoapyrases (ARL-67156) and an inhibitor of the ecto-5'nucleotidase (AOPCP). The effect of glutamate was prevented in the presence of ARL-67156 (Fig. 1g), suggesting that glutamate evoked a release of ATP from Müller cells, which is extracellularly degraded to ADP/adenosine monophosphate (AMP). On the other hand, AOPCP was largely ineffective in preventing the effect of glutamate (Fig. $1 \mathrm{G}$ ), indicating that the majority of adenosine, which is released after stimulation with glutamate, is not formed by extracellular degradation of AMP. Most of the adenosine, which mediates the glutamate effect, is released by facilitated transport, as indicated by the blocking effect of the antagonist of nucleoside transporters, NBTI (Fig. 1h). The data suggest that the swelling-inhibitory effects of glutamate and ATP involve extracellular degradation of ATP to ADP but not of AMP into adenosine.

\section{ATP-evoked calcium responses}

By rapid degradation, ectonucleotidases are suggested to limit the actions of purinergic receptor agonists. In rat retina Müller cells, intracellular calcium responses can be evoked by various purinergic agonists, such as ATP, ADP and uridine triphosphate (UTP) [31]. The predominant P2 receptor subtype that evokes calcium responses in rat Müller cells is the $\mathrm{P}_{2} \mathrm{Y}_{1}$ receptor [31]. ATP-evoked calcium responses in rat Müller cells are restricted to the inner half of the cells, i.e. to the processes that traverse the inner plexiform layer, and the cell endfeet at the inner surface of the retina $[6,32]$. To determine whether extracellular nucleotide metabolism modulates purinergic calcium responses, we recorded the calcium responses in the endfeet of Müller cells in acutely isolated retinal wholemounts upon administration of ATP. ATP-evoked calcium responses were observed in Müller cell endfeet that ensheath the ganglion cell bodies, as well as in glial processes that surround retinal vessels (Fig. 2a). As purinergic receptor agonists do not evoke $\mathrm{P} 2 \mathrm{X}$ receptormediated cation currents in rat Müller cells [33, 34], the calcium responses are suggested to be evoked by activation of P2Y receptors. Inhibition of ectoapyrases by ARL-67156 caused a significant increase in the ATP-evoked calcium responses in the endfeet of Müller cells (Fig. 2b,c). It is concluded that the activity of ectonucleotidases regulates the purinergic receptor-mediated calcium responses in Müller glial cells.

Immunolocalisation of ectonucleotidases

We stained retinal slices and isolated cells to determine the type of ectonucleotidases expressed by rat Müller cells. In 

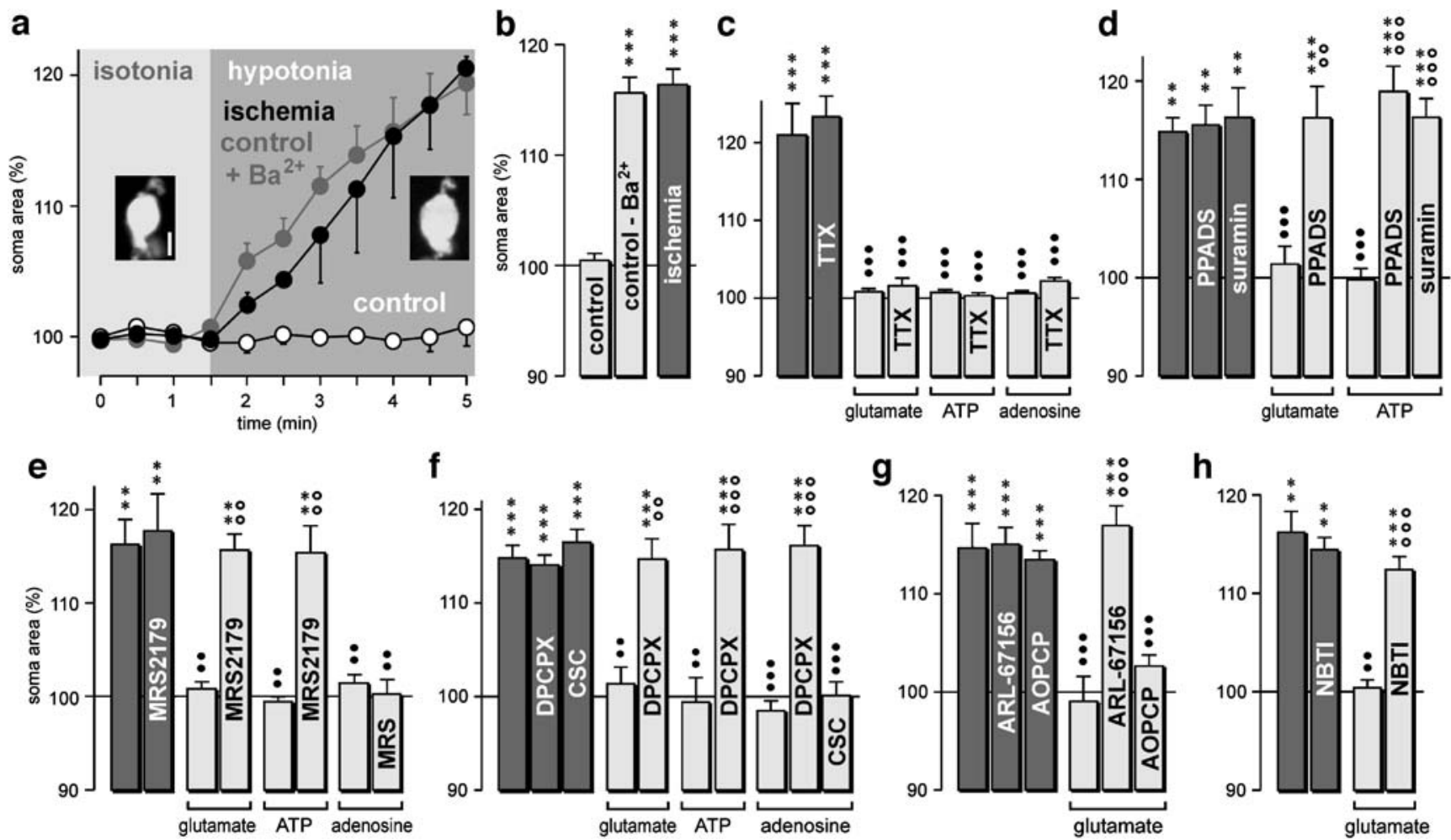

Fig. 1 A purinergic signalling cascade inhibits the osmotic swelling of Müller glial cells. Rat retina slices were perfused with a hypotonic solution $(60 \%$ of control ionic strength), and the cross-sectional area of Müller cell somata was recorded. In (c-h), retinal slices were perfused with a hypotonic solution in the presence of barium chloride $(1 \mathrm{mM})$. a Time-dependent alterations in the soma area of Müller cells in response to a hypotonic solution. Müller cell somata were recorded in a control retina in the absence and presence of barium $(1 \mathrm{mM})$ and in a postischemic retina. Insets: soma of a Müller cell before (left) and during (right) hypotonic exposure. Bar $5 \mu \mathrm{m}$. b Soma area of Müller cells under hypotonic conditions in control and postischemic retinas. c Glutamate, adenosine 5'-triphosphate (ATP) and adenosine, respectively, inhibited the hypotonic swelling of Müller cells somata. This effect remained unaltered in the presence of tetrodotoxin $($ TTX; $1 \mu \mathrm{M})$. d Suramin $(200 \mu \mathrm{M})$ and pyridoxal-phosphate-6-azophenyl-2',4'disulfonic acid (PPADS) $(10 \mu \mathrm{M})$ prevented the swelling-inhibitory effects of glutamate and ATP. e The effects of glutamate and ATP (but not of adenosine) were abolished by the selective blocker of $\mathrm{P} 2 \mathrm{Y}_{1}$ receptors, MRS2179 $(30 \mu \mathrm{M})$. f The antagonist of adenosine A1

receptors, 8-cyclopentyl-1,3-dipropylxanthine (DPCPX) (100 nM), prevented the effects of glutamate, ATP and adenosine. The antagonist of adenosine A2a receptors, 8-(3-chlorostyryl) caffeine (CSC) (200 nM), did not inhibit the effect of adenosine. $\mathbf{g}$ The effect of glutamate involves extracellular metabolism of ATP. The glutamate effect was prevented by the ectoapyrase inhibitor $6-N, N$-diethyl- $D-\beta, \gamma$-dibromomethylene ATP (ARL-67156) $(50 \mu \mathrm{M})$ and remained largely unaltered in the presence of the ecto-5'-nucleotidase inhibitor, adenosine-5'-O- $(\alpha, \beta-$ methylene)-diphosphate (AOPCP) $(250 \mu \mathrm{M})$. hThe swelling-inhibitory effect of glutamate was abolished in the presence of the antagonist of nucleoside transporters, $N$-nitrobenzylthioinosine (NBTI) $(10 \mu \mathrm{M})$. Agonists were coadministered with the hypotonic solution and tested at the following concentrations: glutamate, $1 \mathrm{mM}$; ATP, $10 \mu \mathrm{M}$; adenosine, $10 \mu \mathrm{M}$. Bar diagrams display mean \pm standard error of the mean soma areas, which were measured after a 4-min perfusion of the slices with the hypotonic solution $(n=4-16$ cells per bar). $* * P<0.01$, $* * * P<0.001$ compared with data obtained before osmotic challenge $(100 \%) . \quad{ }^{*} P<0.01, \cdots P<0.001$ compared with data obtained from swollen cells. ${ }^{\circ 0} P<0.05,{ }^{\circ 0} P<0.001$ compared with the agonist effects

retinal slices, immunoreactivity for NTPDase1 was predominantly localised in large blood vessels within the nerve fibre/ganglion cell layers. In addition, faint staining was observed in the microvessels of the inner nuclear layer (arrows in Fig. 3a). Retinal neurons and glial cells, as well as isolated Müller cells (Fig. 3b), were apparently devoid of immunoreactivity for NTPDase1. Müller cells express NTPDase2, as indicated by the glutamine-synthetaseexpressing Müller cell fibres that traverse the inner plexiform layer in retinal slices (filled arrowheads in Fig. 3a), and the immunolabeling of isolated Müller cells
(Fig. 3b). The NTPDase2 protein is localised at the whole plasma membrane of Müller cells, including the membranes of the cell endfeet and somata. In addition to Müller cell fibres, NTPDase2 is expressed in both plexiform (synaptic) layers. Immunoreactivity for ENPP1 was largely absent in the inner retina, whereas a slight staining of Müller cell fibres in the outer retina (unfilled arrowheads in Fig. 3a) was apparent in some (but not all) retinal slices investigated. Müller cells expressed immunoreactivity for the ecto-5'nucleotidase, in particular, at the inner stem processes (filled arrowheads in Fig. 3a) and endfeet, but also at the 


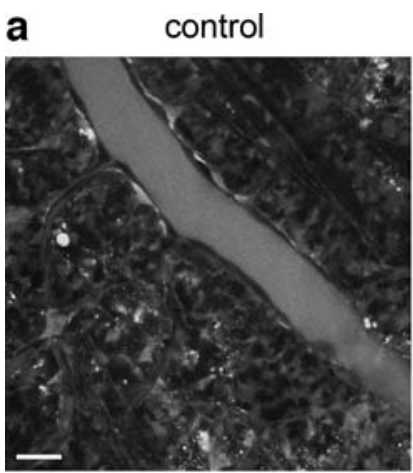

b

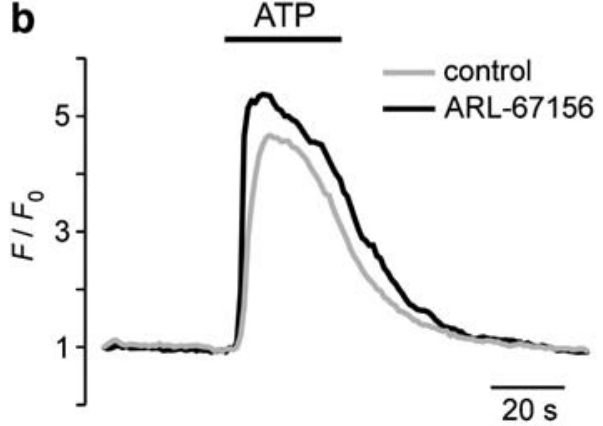

ATP

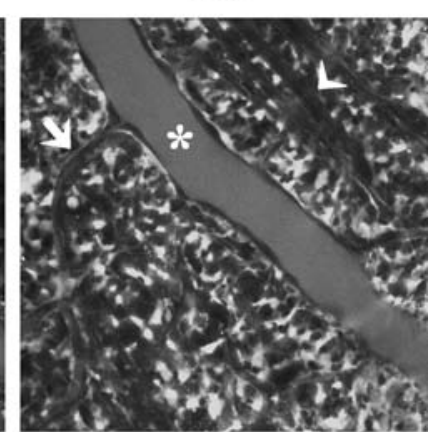

C

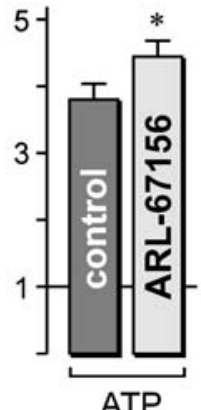

ATP

Fig. 2 Extracellular nucleotide metabolism modulates the adenosine 5'triphosphate (ATP)-evoked calcium responses in rat retina Müller cell endfeet. a Views onto the nerve fibre/ganglion cell layers of a retinal wholemount. The images show the fluorescence of Fluo-4 and were taken before (control) and during the peak calcium response upon bath appplication of ATP $(50 \mu \mathrm{M})$. Asterisk, retinal vein. Arrow, capillary. Arrowhead, nerve fibre bundles. Bar $20 \mu \mathrm{m}$. b Time-dependent changes of the relative fluorescence ratio in Müller cell endfeet. The ATP $(10 \mu \mathrm{M})$-evoked responses were recorded in the absence (control) and presence of 6- $N, N$-diethyl- $D-\beta, \gamma$-dibromomethylene ATP (ARL-67156) $(50 \mu \mathrm{M})$. A fluorescence ratio of one means no change in the cytosolic free calcium level. c Mean ( \pm standard error of the mean) amplitude of the ATP $(10 \mu \mathrm{M})$-evoked calcium responses $(n=38$ and 50 endfeet per bar). ${ }^{*} P<0.05$ compared with the ATP control

outer stem processes (Fig. 3b). In addition, neuronal cell bodies in the ganglion cell and inner nuclear layers may express immunoreactivity for ecto-5'-nucleotidase (Fig. 3a). The data suggest that Müller cells express enzymes for the extracellular degradation of ATP and AMP (NTPDase2 and ecto-5'-nucleotidase).

We found that the inhibition of osmotic Müller cell swelling is mediated by the consecutive activation of $\mathrm{P}_{2} \mathrm{Y}_{1}$ and adenosine A1 receptors (see above). Müller cells express the immunoreactivities for both receptor proteins, which is indicated by the staining of vimentin-positive Müller cell fibres in the inner plexiform layer (filled arrowheads in Fig. 3a). In addition, neuronal cell bodies in the inner nuclear and ganglion cell layers apparently express immunoreactivities for $\mathrm{P}_{2} \mathrm{Y}_{1}$ and $\mathrm{A} 1$ receptors (Fig. 3a). Induction of osmotic swelling is a characteristic feature of Müller cells after transient retinal ischaemia (Fig. 1a,b) [26], which can be inhibited by activation of the purinergic signalling cascade described above [6]. We stained retinal slices that were obtained 3 days after transient retinal ischaemia and found no alteration in the overall localisation of the various enzyme and receptor proteins investigated (Fig. 3c and not shown). An apparent upregulation of the immunoreactivities in the inner retina [ganglion cell layer (GCL), inner plexiform layer (IPL), inner nuclear layer (INL)] may be caused by the strong decrease in thickness of the inner retinal layers (in particular, of the IPL), which is a typical degeneration pattern of rodent retinas after transient ischemia.

\section{Ectonucleotidase histochemistry}

To further identify the nature of retinal ectonucleotidases, we analysed retinal tissues of NTPDase1-deficient and wild-type mice using enzyme histochemistry. When ATP was used as substrate, histochemical staining was observed throughout the retinal tissue of both wild-type and knockout mice, with preferential reaction deposit in the outer retina (Fig. 4a). This suggests that deletion of the NTPDase1 gene has no significant effect on ATPase activity in the retina. Even though the resolution of the enzyme histochemical images is limited due to dispersion of the reaction product, they correspond closely to the immunostaining for NTPDase 2 in the rat retina, except that blood vessels are stained in addition (Fig. 3a). In contrast, when ADP was applied as substrate in wild-type mouse retinas for the same length of time as ATP, staining of the retinal parenchyma was largely abolished. However, strong staining of the vessels was maintained (Fig. 4b). This pattern of enzyme histochemical staining was very similar to the immunohistochemical staining for NTPDase 1 (Fig. 3a). The enzyme histochemical staining at blood vessels for ADP was abolished in retinas of NTPDase1deficient mice (Fig. 4b). No staining of the neural retina was observed when pNPP was applied as a substrate of alkaline phosphatase (Fig. 4c). The pigment epithelium of both wild-type and knockout mice was strongly stained with all substrates used, suggesting that it is rich in nonspecific phosphatases (alkaline phosphatase). No reaction product

Fig. 3 Immunohistochemical localisation of ectonucleotidases and purinergic receptors in rat retina Müller glial cells. a Retinal slices. b Isolated Müller cells. c Slices of postischemic retinas that were obtained from animals 3 days after transient retinal ischemia. The tissues and cells were coimmunostained against the glial-cell-specific proteins vimentin, glutamine synthetase or mouse anti-cellular retinaldehyde-binding protein (CRALBP). Filled arrows, blood vessels. Filled arrowheads, Müller cell fibres in the inner nuclear layer (INL). Unfilled arrows, Müller cell endfeet. Unfilled arrowheads, a, c Müller cell fibres in the outer nuclear layer $(O N L)$ and b Müller cell somata, respectively. GCL ganglion cell layer, $I P L$ inner plexiform layer, $P R S$ photoreceptor segments. Bars $20 \mu \mathrm{m}$ 


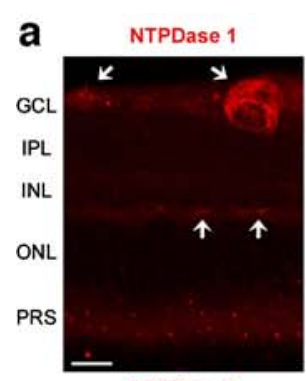

NTPDase 2

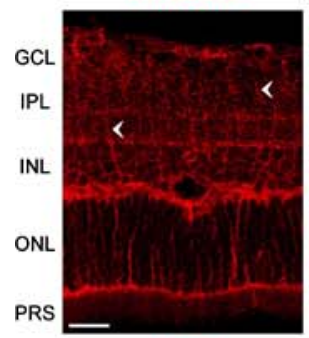

ENPP 1

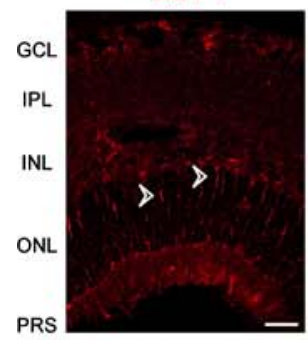

ecto-5'-nucleotidase

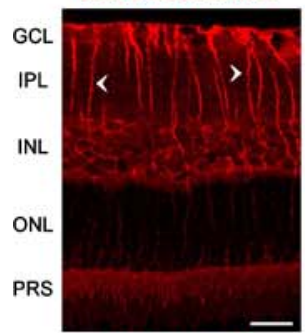

P2Y 1 receptor

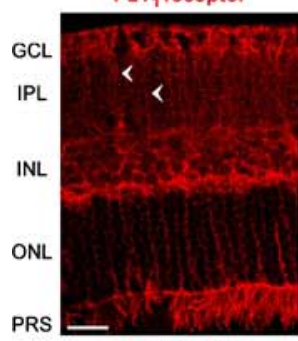

A1 receptor

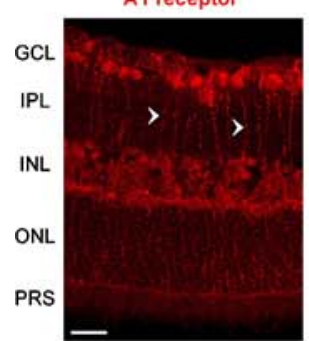

glutamine synthetase

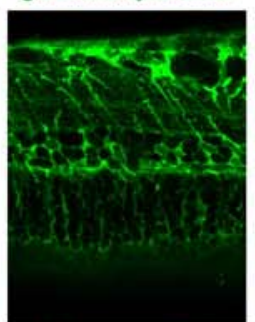

glutamine synthetase

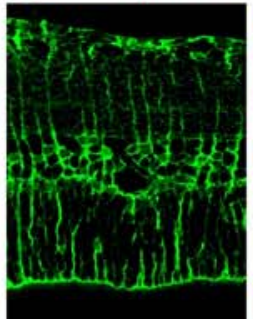

vimentin

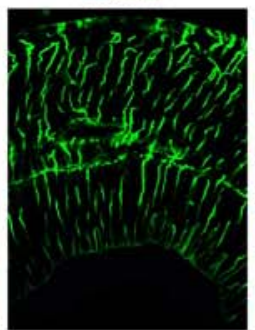

CRALBP

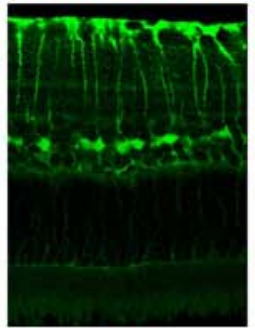

vimentin

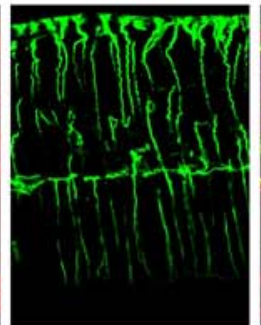

vimentin

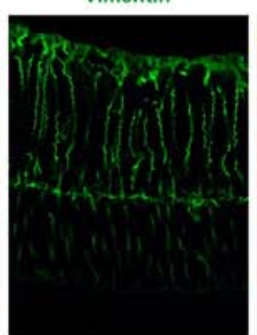

merge

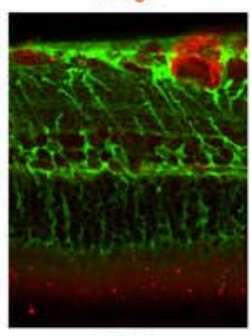

merge

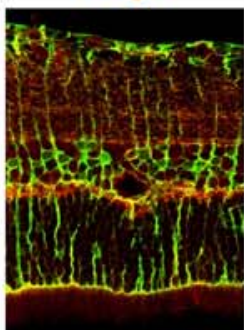

merge

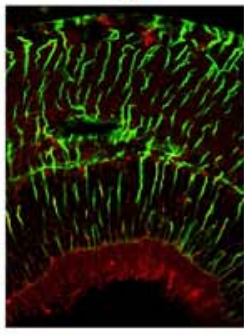

merge

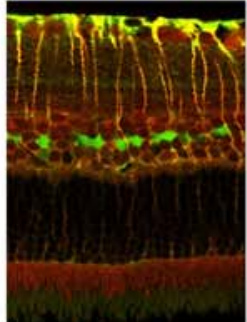

merge

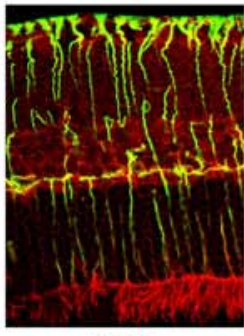

merge

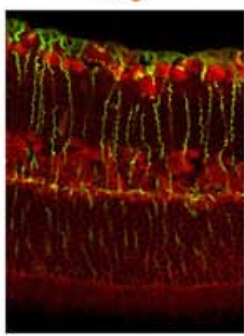

b NTPDase 1

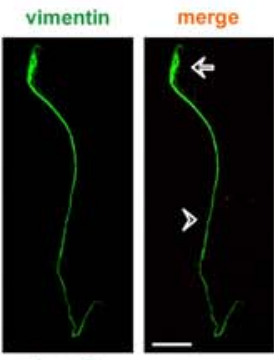

vimentin

merge

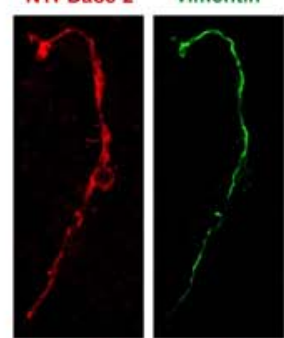

CRALBP

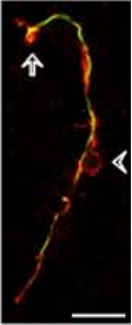

merge

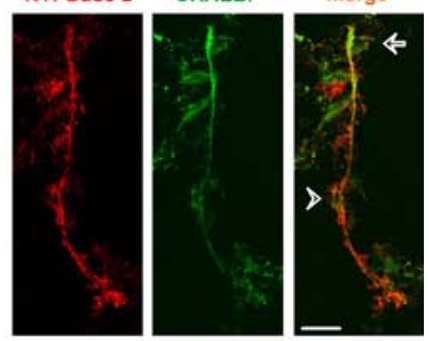

ecto-5'-nucleotidase vimentin

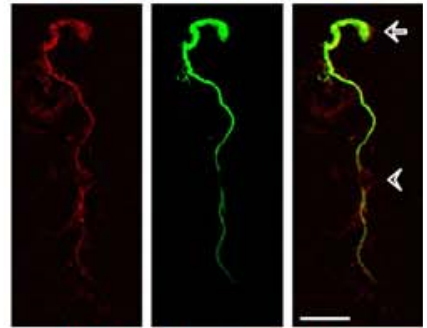

C

NTPDase 2

glutamine synthetase

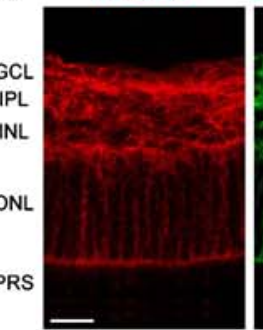

$\mathrm{P}_{2} \mathrm{Y}_{1}$ receptor

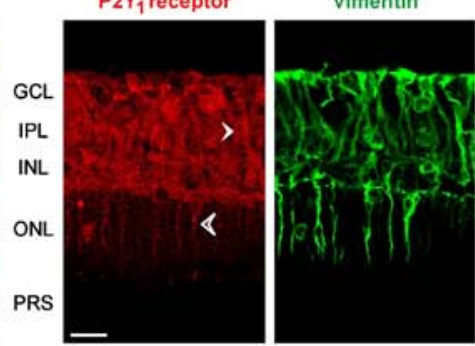


Fig. 4 Enzyme histochemical localisation of ectonucleotidase activity in retinal slices of nucleoside triphosphate diphosphohydrolases (NTPDase1) wild-type $(c d 39+/+)$ and NTPDase1-deficient (cd39-/-) mice. a When adenosine 5'triphosphate (ATP) is used as substrate, the reaction product is distributed throughout the retinal tissue, with most intense staining in the outer plexiform layer. b The use of adenosine 5'diphosphate (ADP) as substrate results in a staining of retinal blood vessels (arrows). This staining is lost in NTPDase1deficient mice. c $p$-nitrophenyl phosphate (pNPP), a substrate of alkaline phosphatase, reveals no retinal staining except of the pigment epithelium. The pigment epithelium has been lost in some sections. GCL ganglion cell layer, $I N L$ inner nuclear layer, $I P L$ inner plexiform layer, $O N L$ outer nuclear layer, $P R S$ photoreceptor segments. Bars $20 \mu \mathrm{m}$

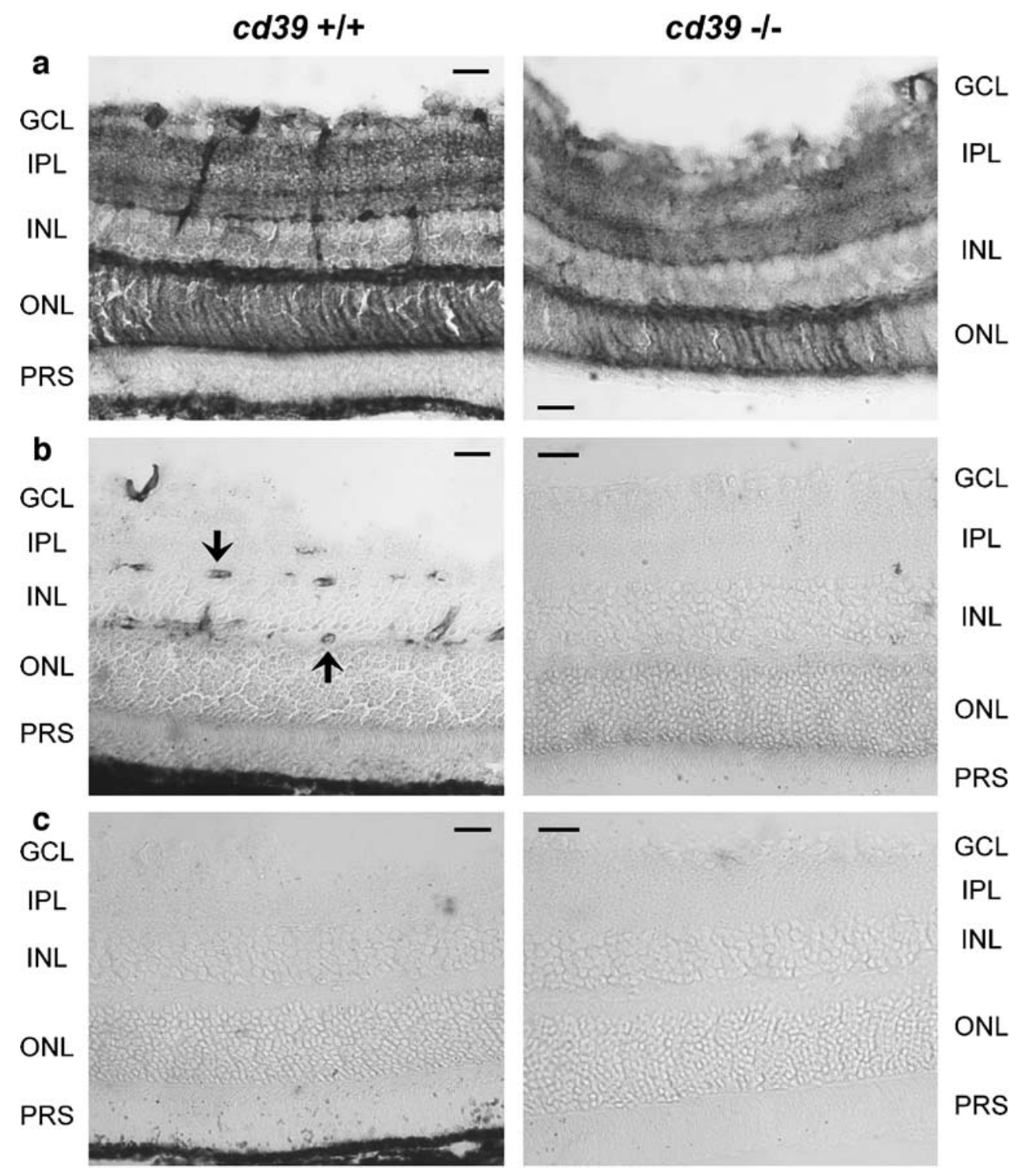

was obtained in the absence of substrates (not shown). The data suggest that NTPDase2 (that has a very high preference for ATP as a substrate, ATP to ADP hydrolysis ratio of $\sim 10: 1$ [35]), is the major ATP-degrading ectonucleotidase of the neural retina, where it is associated with Müller cells. NTPDase1 (that equally hydrolyses ATP and ADP) is restricted to blood vessels, and activity of alkaline phosphatase at physiological $\mathrm{pH}$ is essentially absent. The lack of enzyme histochemical staining for ecto-ADPase in the NTPDase1 knockout mice (Fig. 4b) also suggests the absence of NTPDase3 and NTPDase8.

\section{Discussion}

The data presented here suggest that extracellular ATP metabolism is involved in the autocrine regulation of the
Müller cell volume in response to osmotic stress. Obviously, there is a multilevel signalling cascade resulting in a prevention of cellular swelling under hypoosmotic stress. Whereas we focused on the role of ectonucleotidases in this study, other aspects of this cascade were described in a previous study [6]. One of the first steps is the release of glutamate by retinal neurons, which activates metabotropic glutamate receptors on Müller glial cells [6]. Because a blockade of neuronal activity by tetrodotoxin did not abolish the effect of glutamate, we concluded that neurons are not involved in the cascade downstream from activation of metabotropic glutamate receptors. The pharmacological data presented in Fig. 1 suggest the further course of the cascade. Activation of glial metabotropic glutamate receptors evokes a release of ATP, which is assumed to be metabolised to ADP. This assumption is supported by the finding that the inhibitor of ectoapyrases, ARL-67156, 
prevented the swelling-inhibitory effect of glutamate (Fig. 1g). In addition to the unspecific blockers of P2 receptors, suramin and PPADS, the $\mathrm{P}_{2} \mathrm{Y}_{1}$-specific inhibitor, MRS2179, efficiently suppressed the effects of glutamate and ATP, suggesting that ADP resulting from the action of ectoapyrases activates $\mathrm{P}_{2} \mathrm{Y}_{1}$ receptors on Müller cells. Expression of this receptor subtype on rat Müller cells was demonstrated previously $[31,36]$. The further degradation of ADP to AMP and adenosine does not play a decisive role in the cascade because the blocker of ecto- $5^{\prime}$ nucleotidases, AOPCP, reduced the glutamate effect nonsignificantly. However, adenosine is obviously involved in the cascade. Whereas inhibition of $\mathrm{P}_{2} \mathrm{Y}_{1}$ prevented the effects of glutamate and ATP, it did not alter the action of adenosine. Thus, the effect of adenosine is an event downstream from the activation of glutamate and $\mathrm{P} 2 \mathrm{Y}_{1}$ receptors. Using two different subtype-specific adenosine receptor blockers, we concluded that the adenosine effect is mediated by activation of A1 receptors (Fig. 1f). Because extracellular formation of adenosine is likely to be only a minor source of this nucleoside, the blocker of nucleoside transporters, NBTI, was tested and prevented the effect of glutamate. This suggests that the main part of adenosine is liberated via a transporter. We did not investigate the final steps of the cascade downstream from the activation of A1 receptors. It was concluded from previous data $[6,37]$ that activation of $\mathrm{A} 1$ receptors causes the opening of potassium and chloride channels in the Müller cell membrane; thus, the cells extrude potassium and chloride ions, which is associated with a water efflux that prevents swelling under hypoosmotic conditions. Considering the results presented in this study, the extracellular enzymatic degradation of ATP to ADP might play a crucial role in the purinergic cascade involved in the autocrine regulation of the Müller cell volume.

The activity of ectonucleotidases controls the level of purinergic receptor agonists in the extracellular space. We found that inhibition of ectoapyrases increased the ATPevoked calcium responses in Müller cell endfeet, suggesting that ectonucleotidases control the availability of extracellular ATP that evokes calcium responses predominantly by activation of $\mathrm{P}_{2} \mathrm{Y}_{1}$ receptors [31]. The relatively small effect of the ectoapyrase inhibitor may be explained by the possibility that ATP-evoked ATP release from Müller cells may overwhelm the extracellular degradation of ATP. By rapid inactivation of purinergic signalling, ectonucleotidases may regulate the light-evoked purinergic neuron-to-glia signalling in the retina [32] and the longrange purinergic calcium signalling in the network of retinal glial cells [4].

In a third step of the study, we investigated the expression of enzymes involved in extracellular nucleotide degradation in Müller cells. We found that Müller cells of the rat express immunoreactivities for NTPDase 2 and ecto5 -nucleotidase but not for NTPDase1 and only faintly for ENPP1. The expression of ecto-5'-nucleotidase is in agreement with previous studies [7-9]. The expression of NTPDase 2 by Müller cells is a novel finding and is in agreement with data that showed an expression of NTPDase2 (but not NTPDase1) in distinct glial cells of the brain and peripheral nervous system [16, 17, 19], as well as with a recent study that described a localisation of ecto-ATPase activity at the electron microscopic level in Müller cell membranes (in addition to synaptic membranes) [38]. The enzyme histochemical experiments revealed that NTPDase2 is the major ATP-degrading ectonucleotidase of the neural mouse retina, whereas NTPDase1 activity is restricted to blood vessels and alkaline phosphatase is essentially absent at physiological $\mathrm{pH}$. The data also rule out the possibility of a significant expression of NTPDases 3 and 8 in the retinal parenchyma. Our data confirm previous studies describing an exclusive localisation of NTPDase1-like activity and protein to the vasculature of the neural retina $[14,15]$. According to our data, ATP can be rapidly hydrolysed within the retinal parenchyma, whereas (due to the very low ADPase activity of NTPDase2) ADP will be degraded with a considerable delay. The retinal pigment epithelium apparently expresses the activity of nonspecific phosphatases (alkaline phosphatase) and may also express various other extracellular nucleotide-degradative enzymes [39].

The enzyme histochemical data are in agreement with the results of the cell-swelling experiments indicating that ecto-5'-nucleotidase activity is not involved in the regulation of Müller cell volume, likely due to the absence of the substrate AMP. The small, nonsignificant effect of the 5'nucleotidase inhibitor (Fig. 1g) may reflect the very slow and delayed degradation of ADP to AMP by NTPDase2 [35]. However, further investigations are necessary to confirm the involvement of extracellular nucleotide metabolism in the regulation of Müller cell volume. The nucleotide analogue ARL-67156 has been suggested to inhibit the activities of ectoapyrases in several tissues [40 42]. A recent study using a capillary electrophoresis enzyme assay has shown that ARL-67156 blocks predominantly the activity of recombinant NTPDases 1 and 3 and has only a minor effect on the activity of recombinant NTPDase2 [43]. Our experimental data imply that NTPDase2 was inhibited by ARL-67156 in retinal slices. It remains to be determined whether the functional state of NTPDase2 (such as its oligomeric state [35]) can govern its sensitivity to certain inhibitors or whether ARL-67156 may exert additional effects in intact tissues in situ, such as a blockade of the release of ATP from agonist-stimulated cells [44].

Both extracellular degradation of ATP and the formation of adenosine have been implicated in the suppression of 
neuronal activity in the retina [5]. It has been suggested that ATP released from Müller cells is converted extracellularly to adenosine, which in turn activates A1 receptors in a subset of retinal ganglion cells, resulting in cellular hyperpolarisation [5]. The present results indicate that both neuronal cells and Müller cells express ecto-5'-nucleotidase, that forms adenosine from AMP. On the other hand, we found that only the activity of ecto-ATPases (and not of the ecto-5'-nucleotidase) is implicated in the regulation of Müller cell volume and that comparable ecto-ADPase activity (which may deliver AMP as the substrate of ecto-5'nucleotidase) is absent from the retinal parenchyma. The reason for the discrepancy with previously published data [5] is unclear and remains to be solved in future investigations. It cannot be ruled out that pharmacological modulation of the glial purinergic signalling may also indirectly alter neuronal activity via alterations of the glial cell volume and, therefore, of the extracellular space volume. Moreover, differences in the local microenvironment and the spatial relationship of ectonucleotidases, purinergic receptors and transporter molecules may explain some of the different findings.

In summary, we found that NTPDase2 is the major ATPdegradative ectonucleotidase in the parenchyma of rodent retinas, whereas NTPDase1 is expressed solely in retinal vessels. The activity of NTPDase 2 is suggested to be implicated in the regulation of the Müller cell volume under osmotic stress conditions. This regulation is likely mediated by extracellular degradation of released ATP and subsequent transporter-mediated release of adenosine. The activity of NTPDase2 is also involved in the regulation of ATP-evoked calcium responses in Müller cells; this may have functional importance for the purinergic neuron-to-glia signalling observed in response to light stimulation [32]. Additional investigations are required to determine the functional roles of NTPDase2 and ecto-5'-nucleotidase in the reciprocal signalling between neurons and glia in the retina.

Acknowledgements This work is gratefully dedicated to Peter Illes for his engagement to push forward the research of purinergic signalling at the University of Leipzig. We thank Klaus Hammer for expert support with the enzyme histochemistry. This work was supported by grants from the BMBF to the Translational Center for Regenerative Medicine Leipzig (1113MB), from the Interdisziplinäres Zentrum für Klinische Forschung (IZKF) at the University of Leipzig Faculty of Medicine (C35, Z10), and the Deutsche Forschungsgemeinschaft (GRK 1097/1, RE 849/12, to A.R.; FOR 748, ZI 140/18-1 to H.Z.).

\section{References}

1. Haydon PG (2001) Glia: listening and talking to the synapse. Nat Rev Neurosci 2:185-193

2. Nedergaard M, Takano T, Hansen AJ (2002) Beyond the role of glutamate as a neurotransmitter. Nat Rev Neurosci 3:748-755
3. Newman EA (2003) New roles for astrocytes: regulation of synaptic transmission. Trends Neurosci 26:536-542

4. Newman EA (2001) Propagation of intercellular calcium waves in retinal astrocytes and Müller cells. J Neurosci 21:2215-2223

5. Newman EA (2003) Glial cell inhibition of neurons by release of ATP. J Neurosci 23:1659-1666

6. Uckermann O, Wolf A, Kutzera F et al (2006) Glutamate release by neurons evokes a purinergic inhibitory mechanism of osmotic glial cell swelling in the rat retina: activation by neuropeptide Y. J Neurosci Res 83:538-550

7. Kreutzberg GW, Hussain ST (1982) Cytochemical heterogeneity of the glial plasma membrane: 5'-nucleotidase in retinal Müller cells. J Neurocytol 11:53-64

8. Hussain ST, Baydoun EA (1985) Cytochemical localization of 5'nucleotidase in the frog (Rana pipiens) retina. A histochemical and cytochemical study. J Histochem Cytochem 33:1067-1072

9. Braun N, Brendel P, Zimmermann H (1995) Distribution of 5'nucleotidase in the developing mouse retina. Brain Res Dev Brain Res 88:79-86

10. Lutty GA, Merges C, McLeod DS (2000) 5' nucleotidase and adenosine during retinal vasculogenesis and oxygen-induced retinopathy. Invest Ophthalmol Vis Sci 41:218-229

11. Zimmermann H (1992) 5'-Nucleotidase: molecular structure and functional aspects. Biochem J 285:345-365

12. Zimmermann H (2001) Ectonucleotidases: some developments and a note on nomenclature. Drug Dev Res 52:44-56

13. Zimmermann H (2000) Extracellular metabolism of ATP and other nucleotides. Naunyn Schmiedeberg's Arch Pharmacol 362:299-309

14. Lutty GA, McLeod DS (1992) A new technique for visualization of the human retinal vasculature. Arch Ophthalmol 110:267-276

15. McLeod DS, Hasegawa T, Prow T, Merges C, Lutty G (2006) The initial fetal human retinal vasculature develops by vasculogenesis. Dev Dyn 235:3336-3347

16. Braun N, Sevigny J, Robson SC et al (2000) Assignment of ectonucleoside triphosphate diphosphohydrolase-1/cd39 expression to microglia and vasculature of the brain. Eur J Neurosci 12:4357-4366

17. Braun N, Sevigny J, Mishra SK et al (2003) Expression of the ecto-ATPase NTPDase2 in the germinal zones of the developing and adult rat brain. Eur J Neurosci 17:1355-1364

18. Shukla V, Zimmermann H, Wang L et al (2005) Functional expression of the ecto-ATPase NTPDase2 and of nucleotide receptors by neuronal progenitor cells in the adult murine hippocampus. J Neurosci Res 80:600-610

19. Braun N, Sevigny J, Robson SC, Hammer K, Hanani M, Zimmermann H (2004) Association of the ecto-ATPase NTPDase2 with glial cells of the peripheral nervous system. Glia 45:124-132

20. Rebbe NF, Tong BD, Finley EM, Hickman S (1991) Identification of nucleotide pyrophosphatase/alkaline phosphodiesterase I activity associated with the mouse plasma cell differentiation antigen PC-1. Proc Natl Acad Sci USA 88:5192-5196

21. Asensio AC, Rodriguez-Ferrer CR, Castaneyra-Perdomo A, Oaknin S, Rotllan P (2007) Biochemical analysis of ectonucleotide pyrophosphatase phosphodiesterase activity in brain membranes indicates involvement of NPP1 isoenzyme in extracellular hydrolysis of diadenosine polyphosphates in central nervous system. Neurochem Int 50:581-590

22. Ohkubo S, Kimura J, Matsuoka I (2000) Correlation between adenine nucleotide-induced cyclic AMP elevation and extracellular adenosine formation in NG108-15 cells. Jpn J Pharmacol 84:325-333

23. Ohkubo S, Kumazawa K, Sagawa K, Kimura J, Matsuoka I (2001) $\beta, \gamma$-Methylene ATP-induced cAMP formation in C6Bu-1 
cells: involvement of local metabolism and subsequent stimulation of adenosine A2B receptor. J Neurochem 76:872-880

24. Enjyoji K, Sévigny J, Lin Y et al (1999) Targeted disruption of $c d 39 / A T P$ diphosphohydrolase results in disordered hemostasis and thromboregulation. Nat Med 5:1010-1017

25. Uckermann O, Iandiev I, Francke M et al (2004) Selective staining by vital dyes of Müller glial cells in retinal wholemounts. Glia 45:59-66

26. Pannicke T, Iandiev I, Uckermann O et al (2004) A potassium channel-linked mechanism of glial cell swelling in the postischemic retina. Mol Cell Neurosci 26:493-502

27. Wurm A, Pannicke T, Iandiev I, Wiedemann P, Reichenbach A, Bringmann A (2006) The developmental expression of $\mathrm{K}^{+}$ channels in retinal glial cells is associated with a decrease of osmotic cell swelling. Glia 54:411-423

28. Ralevic V, Burnstock G (1998) Receptors for purines and pyrimidines. Pharmacol Rev 50:413-492

29. Vöhringer C, Schäfer R, Reiser G (2000) A chimeric rat brain $\mathrm{P} 2 \mathrm{Y}_{1}$ receptor tagged with green-fluorescent protein: high-affinity ligand recognition of adenosine diphosphates and triphosphates and selectivity identical to that of the wild-type receptor. Biochem Pharmacol 59:791-800

30. Mishra SK, Braun N, Shukla V et al (2006) Extracellular nucleotide signalling in adult neural stem cells: synergism with growth factormediated cellular proliferation. Development 133:675-684

31. Li Y, Holtzclaw LA, Russell JT (2001) Müller cell $\mathrm{Ca}^{2+}$ waves evoked by purinergic receptor agonists in slices of rat retina. $\mathrm{J}$ Neurophysiol 85:986-994

32. Newman EA (2005) Calcium increases in retinal glial cells evoked by light-induced neuronal activity. J Neurosci 25:5502-5510

33. Felmy F, Pannicke T, Richt JA, Reichenbach A, Guenther E (2001) Electrophysiological properties of rat retinal Muller (glial) cells in postnatally developing and in pathologically altered retinae. Glia 34:190-199
34. Bringmann A, Pannicke T, Moll V et al (2001) Upregulation of $\mathrm{P}_{2} \mathrm{X}_{7}$ receptor currents in Müller glial cells during proliferative vitreoretinopathy. Invest Ophthalmol Vis Sci 42:860-867

35. Failer BU, Aschrafi A, Schmalzing G, Zimmermann H (2003) Determination of native oligomeric state and substrate specificity of rat NTPDase1 and NTPDase2 after heterologous expression in Xenopus oocytes. Eur J Biochem 270:1802-1809

36. Pannicke T, Weick M, Uckermann O et al (2001) Electrophysiological alterations and upregulation of ATP receptors in retinal glial Müller cells from rats infected with the Borna disease virus. Glia 35:213-223

37. Skatchkov SN, Eaton MJ, Shuba YM et al (2006) Tandem-pore domain potassium channels are functionally expressed in retinal (Müller) glial cells. Glia 53:266-276

38. Puthussery T, Fletcher EL (2007) Neuronal expression of $\mathrm{P} 2 \mathrm{X}_{3}$ purinoceptors in the rat retina. Neuroscience 146:403-414

39. Reigada D, Lu W, Zhang $X$ et al (2005) Degradation of extracellular ATP by retinal pigment epithelium. Am J Physiol 289:C617-C624

40. Crack BE, Pollard CE, Beukers MW et al (1995) Pharmacological and biochemical analysis of FPL 67156, a novel, selective inhibitor of ecto-ATPase. Br J Pharmacol 114:475-481

41. Kennedy C, Westfall TD, Sneddon P (1996) Modulation of purinergic neurotransmission by ecto-ATPase. Semin Neurosci 8:195-199

42. Dowd FJ, Li LS, Zeng W (1999) Inhibition of rat parotid ectoATPase activity. Arch Oral Biol 44:1055-1062

43. Iqbal J, Vollmayer P, Braun N, Zimmermann H, Müller CE (2005) A capillary electrophoresis method for the characterization of ecto-nucleoside triphosphate diphosphohydrolases (NTPDases) and the analysis of inhibitors by in-capillary enzymatic microreaction. Purinergic Signal 1:349-358

44. Bodas E, Aleu J, Pujol G, Martin-Satué M, Marsal J, Solsona C (2000) ATP crossing the cell plasma membrane generates an ionic current in Xenopus oocytes. J Biol Chem 275:20268-20273 